\title{
Effect of heat and pressure processing on DNA fragmentation and implications for the detection of meat using a real-time polymerase chain reaction
}

\author{
H. HIRD ${ }^{1}$, J. CHISHOLM ${ }^{1}$, A. SANCHEZ ${ }^{2}$, M. HERNANDEZ $^{3}$, R. GOODIER ${ }^{4}$, \\ K. SCHNEEDE , C. BOLTZ ${ }^{5} \&$ B. POPPING ${ }^{6}$
}

${ }^{1}$ Central Science Laboratory, York YO41 1LZ, UK, ${ }^{2}$ Instituto de Investigaciones Marinas, 36208 Vigo, Spain, ${ }^{3}$ Instituto Tecnolo'gico Agrario de Castilla y Leo'n, E-47071 Valladolid, Spain, ${ }^{4}$ BioReliance, Invitrogen bioservices, Glasgow G20 OXA, UK, ${ }^{5}$ Eurofins WEJ, Hamburg D-21079, Germany, and ${ }^{6}$ Eurofins Scientific Group, Pocklington YO42 2JY, UK

(Received 27 June 2005; revised 25 January 2006; accepted 26 January 2006)

Abstract

The design of real-time polymerase chain reaction (PCR) assays for the detection of meat in processed products has focused on using small amplicons, often to the detriment of specificity. However, the relationship between amplification rates and the amplicon size for processed meat products has yet to be determined. To investigate this relationship, real-time PCR assays were designed to give a series of amplicons of increasing size. These assays were then used to assess amplification rates, in relation to amplicon size, in processed meat matrices. Although the most sensitive assays were those that used the smallest amplicons, amplification was still observed using amplicons of 351 base pairs for highly processed samples. It was found, therefore, that although in general, amplicons should be as small as possible, larger amplicons give efficient amplification and that small amplicons should not be chosen if they compromise assay specificity.

Keywords: Real-time polymerase chain reaction, heat, pressure, processing, meat, DNA, detection

Introduction

There are many reports outlining the detection of meat in food in the literature. From these reports, DNA appears to be the analyte of choice and real-time polymerase chain reaction (PCR) the most appropriate analytical tool. However, there are few reports axamining the design and optimization of real-time PCR assays, although many studies cite various significant parameters that must be considered during the design process for efficient amplification and therefore sensitive assays.

Amplification efficiency is a measure of how close the amplification rates are to theorical doubling of template DNA with each cycle. Amplification rates are affected by many factors, amongst which 
include the number of target molecules, the sequence of the target, the buffer and thermostable polymerase, the cycling conditions, the primer sequence and the presence of inhibitors in the sample. One of the most cited parameters, from There are many reports outlining the detection of the work of Meyer et al. (1993), is amplicon size, meat species in food in the literature. From these since this group reported that the average size of reports, DNA appears to be the analyte of choice and DNA fragments following heat and pressure procesreal-time polymerase chain reaction (PCR) the most sing was approximately 300 base pairs (bp). appropriate analytical tool. However, there are few Consequently, most systems for the detection of reports examining the design and optimization of DNA from highly processed matrices used amplireal-time PCR assays, although many studies cite cons of approximately 300 bp (Bottero et al. 2003; various significant parameters that must be considFrezza et al. 2003; Tartaglia et al. 1998) and the ered during the design process for efficient amplifineed for gel visualization meant that the amplicons cation and therefore sensitive assays. were usually over $130 \mathrm{bp}$.

Amplification efficiency is a measure of how close The advent of real-time PCR has allowed the the amplification rates are to the theoretical doubling development of assays that use very small amplicons, of template DNA with each cycle. Amplification and the size of amplicon has, in some studies, rates are affected by many factors, amongst which appeared to override all other considerations, include the number of target molecules, the compromising assay specificity in some instances sequence of the target, the buffer and thermostable (Brodmann and Moor 2003; Dooley et al. 2004; polymerase, the cycling conditions, the primer Mendoza-Romero et al. 2004). Brodmann and Moor designed assays for the smallest possible amplicons to ensure amplification of highly processed feeds. However, using amplicons of 66 and 76 bp there was only intermittent detection of samples of cow bone meal. These primers were also shown to cross-amplify red and roe deer, moose, sheep and pig DNA. Similarly, ruminant specific assays, using an amplicon of $88 \mathrm{bp}$, were shown to cross-amplify with the non-ruminants pig, horse, chicken and turkey (Mendoza-Romero et al. 2004). The interpretation of assays that cross-amplify is problematic since it is unclear whether there has been detection of the target or the cross-amplifying species.

The objective of the present study was to extend the current knowledge for the design and optimization of real-time PCR assays for the detection of meats in food products. Experiments were designed to elucidate the effect of amplicon size on the sensitivity of real-time PCR assays when applied to heat and pressure-processed matrices. Real-time PCR assays were designed for turkey, where one primer remained constant and was paired with a series of alternative primers to give amplicons of increasing size. This primer series was used on raw turkey template DNA to determine the rate of amplification. We went on to study the effect of processing on amplification rates using 
preparations of meat that had been boiled, baked, canned or autoclaved. Finally, the turkey primer series was applied to the detection of turkey in poultry meal to illustrate the effect of amplicon size on the detection of highly processed samples. The data presented herein should allow a greater flexibility during the design of real-time PCR assays, leading to protocols for the detection of meats in food products with increased specificity.

Materials and methods

\section{Meat samples and processing}

Authentic meat samples were sourced from local suppliers and held at 4C until processing. Samples were homogenized in a food blender and aliquots processed or left raw. Boiled samples were sealed in plastic pouches and boiled in water for 2 and 32 min. Baked samples were cooked in an oven at 180C for 2 and 32 min. Canned samples were processed in a Mather and Platt vertical retort, at 121C for $40 \mathrm{~min}$ to achieve a sterilizing (F0) value of at least 7.6 and autoclaved samples were autoclaved at 121C for $15 \mathrm{~min}$. Raw, boiled, baked and autoclaved samples were frozen at 20C; canned samples were stored at room temperature until DNA extraction.

DNA extraction from meat mixtures

DNA was extracted from meat matrices using a modified version of the Wizard DNA extraction Kit (Promega, Southampton, UK). Briefly, $5 \mathrm{~g}$ of each sample were incubated at 65C in $10 \mathrm{ml}$ CTAB buffer (2\% (w/w) cetyl trimethyl ammonium bromide (CTAB), 20 mM EDTA, $0.1 \mathrm{M}$ Tris[hydroxymethyl]aminomethane (Tris-base) and $1.4 \mathrm{M}$ sodium chloride (pH 8) containing $80 \mathrm{ng}$ $\mathrm{ml}^{1}$ proteinase $\mathrm{K}$ (Sigma). After $2 \mathrm{~h}$, the samples were vortexed for $10 \mathrm{~s}$ and centrifuged for $10 \mathrm{~min}$ at $4000 \mathrm{~g}$. A total of $1 \mathrm{ml}$ of the supernatant was combined with $800 \mathrm{ml}$ chloroform, vortexed briefly and centrifuged for $10 \mathrm{~min}$ at $14000 \mathrm{~g}$. A total of $500 \mathrm{ml}$ of the aqueous phase was combined with 1 ml Wizard DNA resin and incubated for $5 \mathrm{~min}$ at room temperature. The DNA was then purified exactly according to the manufacturers' instructions and eluted with $100 \mathrm{ml}$ Tris EDTA buffer previously heated to $65 \mathrm{C}$.

Design of the TaqMan ${ }^{2}$ primer and probe sets

The primers and probe sets were designed using the mitochondrial cytochrome b gene for turkey using Primer Express software (v.1.5). The primers were expected to have the following specific qualities: to produce an amplicon at target sizes; to have the 30 end of the primer as close as possible to the probe without overlapping it; to have a Tm between 58 and 60C; to contain 20-80\% G or C, to be between 9 and 40 bases long, and to have a maximum of $2 \mathrm{G}$ or $\mathrm{C}$ bases within the last five 
residues of the 30 end. Primer Express design parameters were adapted to achieve primer design. The probe was designed to have a $\mathrm{Tm}=10^{\circ} \mathrm{C}$ higher than that of the primers, to contain between 20 and $80 \%$ G or C, to be between 9 and 40 bases long, have a T, C or A at the 50 end, and to contain more C’s than G's (Oehlmann 1999). Additionally, primers were designed to be mismatched at the 30 position to negate spurious amplification with other species of farm animals and humans (Hird et al. 2003). The turkey primers were truncated at the 50 end until specific (Hird et al. 2004). Probes were 50-labelled with 6-carboxyfluorescein (FAM) and 30-labelled with the fluorescent quencher dye 6carboxytetramethylrhodamine (TAMRA). Primers and probes were purchased from Applied Biosystems (Warrington, UK) (Table I).

\section{TaqMan $^{\bullet}$ assay conditions}

Each real-time PCR reaction contained 1TaqMan ${ }^{\mathrm{TM}}$ buffer A, $25 \mathrm{mM} \mathrm{MgCl2}$ solution, 0.625 units AmpliTaq Gold DNA polymerase (Applied Biosystems) and $0.5 \mathrm{mM}$ of each deoxynucleotide triphosphate (Sigma) in $25 \mathrm{ml}$. Primers were used at $900 \mathrm{nM}$ and probes at $200 \mathrm{nM}$. DNA template was diluted in water and $5 \mathrm{ml}$ of either DNA or water, as a negative control, were added per reaction. Reactions were assembled in Axygen thin-walled 96-well plates with optical caps and run on ABI Prism 7700 or 7900 sequence detection systems with the following thermal cycling protocol: 50C for $2 \mathrm{~min}$, 95C for $10 \mathrm{~min}$ followed by 40 cycles of 95C for $15 \mathrm{~s}$ and 60C for $1 \mathrm{~min}$.

Results and discussion

Effect of amplicon length on amplification rates

It is known that food heat and pressure processing can result in DNA fragmentation, which can, in turn, have implications on the sensitivity of DNAbased analyses. Real-time PCR primers and probe systems were designed that would enable us to determine the degree of DNA fragmentation after processing by using amplicons of different lengths. The reverse primer remained constant (anchored in position) and was paired with a series of eight alternative forward primers, giving amplicons of increasing size (81, 116, 240, 317, 351, 427, 470 and 565 bp) (Figure 1).

Primers were identified to be as close as possible to target sizes, e.g. an amplicon length of $120 \mathrm{bp}$ was not possible for this region of turkey sequence and therefore a primer was designed that would give an amplicon as close to $120 \mathrm{bp}$ as possible. Therefore, the design of the primers was largely dependent upon the sequence. Additionally, the Primer Express design parameters were relaxed for amplicons above 150 bp to allow the design of primers giving amplicons larger than 150 bp the usual upper limit for real-time amplicons (Table I). 
These primer sets were then used to investigate the effect of amplicon length on amplification rates using raw template DNA (Figure 2). An increase in CT (the threshold cycle), indicating a reduction in amplification rate was observed with increasing amplicon size for the turkey primer series. Amplicons up to $240 \mathrm{bp}$ had similar amplification rates, whereas for amplicon sizes above $240 \mathrm{bp}$, the increases in CT were more marked, particularly between amplicons of 427 and 565 bp where there was an increase of 5 CT.

The results are in accordance with the technical information supplied by Applied Biosystems. The reactions of Taq polymerase are catalysed by magnesium, and the enzyme has a large tolerance for magnesium concentration with small amplicons. Amplicon sizes below 150 bp are recommended since the magnesium concentration can remain constant. However, the data presented herein suggests that amplicons above 150 bp can also support efficient amplification, since there was a rise of only 1.5 CT when the amplicon size was increased to over $300 \mathrm{bp}$. These data support the view that for abundant template molecules amplicon size should be kept below 150 bp. However, for sequences that prove difficult for primer and probe design, amplicon sizes above 150 bp may also support efficient amplification.

Assessment of the degree of DNA fragmentation during processing

To investigate the effect of heat and pressure processing on the availability of template molecules, the turkey primer series was used to amplify DNA that had been extracted from turkey muscle which had been subjected to the following six different cooking conditions: light boiling, heavy boiling, light baking, heavy baking, canning and autoclaving. The DNA was extracted from duplicate samples, the DNA concentration adjusted to $17 \mathrm{ng} \mathrm{ml}^{1}$ and then $85 \mathrm{ng} /$ reaction were analysed using the real-time PCR primer series for turkey (Figure 3).

In general terms, there was no difference in the amplification rates of DNA extracted from samples that had been boiled or baked in comparison with DNA from raw samples for amplicons of 81, 116, 351 or $565 \mathrm{bp}$. This indicated that the fragmentation of the DNA had not affected amplification for amplicons up to 565 bp since the amplification rates were roughly similar to those of raw samples, regardless of the boiling or baking conditions. This also indicated that amplicons of 351 and 565 bp can amplify template DNA extracted from boiled or baked samples efficiently.

There was a statistically significant increase in the CT returned for the canned and autoclaved samples for all sizes of amplicons. This difference became more pronounced with increasing amplicon size, where amplification of DNA extracted from the autoclaved sample returned a value 1.41 CT greater than amplification of the DNA extracted from the raw sample with the 81 bp primer 
set compared with an increase of 10 CT for the 351 bp primer set and a complete failure for the 565 bp primer set. A similar trend was observed for the canned samples where amplification rates decreased significantly with increasing amplicon length.

Fragmentation of DNA was only observed when the meats were canned or autoclaved, with little or no fragmentation observed when the meats were cooked in the absence of pressure. The effect of DNA fragmentation on PCR-based systems is twofold. First, when DNA fragments, the available template molecules are reduced when a template molecule is cleaved. Second, small DNA fragments are not extracted with the same efficiency as larger template molecules by some DNA extraction systems, particularly for extraction techniques that use DNA precipitation, since small fragments of DNA do not precipitate as easily as large fragments.

In this study, the effect of DNA extractability from heat and pressure-processed matrices on amplification rates may have contributed to the increased CT values observed in the canned and autoclaved samples, although this is an unlikely cause for the increased CT since there was little effect of canning and autoclaving on the amplification rate of the 81 bp amplicon. Had DNA extractability from the canned and autoclaved samples been a major contributor to the increased CT returned for amplicons of 351 and 565 bp, one would have also observed a significant increase in the CT values returned for amplicons of 81 and 116 bp. These data indicated that canning and autoclaving affected the fragmentation of the DNA, rather than the DNA extractability.

To illustrate further the implication of amplicon size on the detection of meats in a mixture, the turkey primer series was used to analyse a sample of poultry meal containing approximately $15 \%$ turkey. Meal of this type is heated to $100 \mathrm{C}$ for $60 \mathrm{~min}$ in the absence of pressure, where the final temperature reached is $120 \mathrm{C}$ resulting in fragmented DNA. Since only a proportion of the sample was derived from turkey, the raw data are to show the effect of amplicon size on the rates of amplification and are not for direct comparison. Nevertheless, DNA fragmentation was evident in this sample since the CT value differential between the raw and processed samples increased with increasing amplicon size, indicating the fragmentation of the target DNA (Figure 4). However, it should be noted that an amplicon of 351 bp was still detectable with a CT of 33.87 in this processed sample.

\section{Conclusions}

Overall, the study showed that the degree of DNA fragmentation correlated with the degree of heat and pressure processing and that this could be assessed using multi-primer assays. DNA fragmentation was minimal for samples that had been baked or boiled, and turkey DNA extracted from autoclaved samples was easily detectable even using amplification systems spanning $351 \mathrm{bp}$. The study proved that amplification rates were not compromised for amplicons of more than $150 \mathrm{bp}$, 
and that in future, the design of real-time PCR assays could afford a greater focus on achieving specificity using a less stringent approach to limiting amplicon size.

\section{References}

Bottero MT, Dalamasso A, Nucera D, Turi RM, Rosati S, Squadrone S, Goria M, Civera T. 2003. Development of a PCR assay for the detection of animal tissues in ruminant feed. Journal of Food Protection 66:2307-2312.

Brodmann PD, Moor D. 2003. Sensitive and semiquantitative TaqMan ${ }^{\text {TM }}$ real-time polymerase chain reaction systems for the detection of beef (Bos taurus) and the detection of the family Mammalia in food and feed. Meat Science 65:599-607.

Dooley JJ, Paine KE, Garrett SD, Brown HM. 2004. Detection of meat species using TaqMan realtime PCR assays. Meat Science 68:431-438.

Frezza D, Favaro M, Vaccari G, Von-Holst C, Giambra V, Anklam E, Bove D, Battaglia PA, Agrimi U, Brambilla G, Ajomone-Marsen P, Tartaglia M. 2003. A competitive polymerase chain reactionbased approach for the identification and semiquantification of mitochondrial DNA in differently heat-treated bovine meat and bone meal. Journal of Food Protection 66:103-109.

Hird H, Goodier R, Hill M. 2003. Rapid detection of chicken and turkey in heated meat products using the polymerase chain reaction followed by amplicon visualisation with vistra green. Meat Science 65:1117-1123.

Hird H, Goodier R, Schneede K, Boltz C, Chisholm J, Lloyd J, Popping B. 2004. Truncation of oligonucleotide primers confers specificity on real time-PCR assays for food authentication. Food Additives and Contaminants 21:1035-1040.

Mendoza-Romero L, Verkaar ELC, Savelkoul PH, Catsburg A, Aarts HJM, Buntjer JB, Lenstra JA. 2004. Real-time PCR detection of ruminant DNA. Journal of Food Protection 67:550-554.

Meyer R, Candrian U, Luthy J. 1993. Detection of pork in heated meat products by the polymerase chain reaction. Journal of AOAC International 77:617-622.

Oehlmann R. 1999. Primer Express manual 1.0. PE Applied Biosystems, Foster City, USA.

Tartaglia M, Saulle E, Pestalozza S, Morrelli L, Antonucci G, Battaglia P. 1998. Detection of bovine mitochondrial DNA in ruminant feeds: A molecular approach to test for the presence of bovinederived materials. Journal of Food Protection 61:513-518. 